\title{
Report of Banbury Summit meeting on training of physicians in medical genetics, October 20-22, 2004
}

\author{
Bruce R. Korf, $M D, P h D^{1}$, Gerald Feldman, $M D, P h D^{2}$, and Georgia L. Wiesner, $M D^{3}$
}

\begin{abstract}
Although we have entered an era in which genetics is becoming increasingly integrated into all areas of medicine, the number of physicians choosing to practice the primary specialty of medical genetics is declining. Medical genetics has existed as a distinct specialty for over 20 years, and for most of this time, medical geneticists have focused on the care of individuals with rare disorders. Now, as the scope of practice is poised for expansion into more common disorders, there is concern that insufficient numbers of medical geneticists are being trained, and that current training does not provide adequate preparation for the next generation to lead this expansion. In an effort to begin to explore these concerns, a meeting was held at the Banbury Conference Center in Cold Spring Harbor, Long Island, New York, October 20-22, 2004. Participants included representatives of major genetics professional organizations in the United States and Canada (see Appendix 1 and 2). Each of the representatives then reported back to their respective organization the results of this conference, and the organizations plan to work together to continue the process. This document will summarize the major discussion points and conclusions from the meeting.
\end{abstract}

\section{FOCUS OF THE MEETING}

The Banbury Summit meeting was arranged specifically to consider ways of increasing the number of physicians who receive training in medical genetics. The practice of medical genetics is not limited to physicians, however. Genetic counselors and laboratory geneticists play key roles, and any discussion about the future of medical genetics must include these disciplines. Medical genetics also intersects with many other medical specialties, including pediatrics, obstetrics and gynecology, family medicine, internal medicine, and many others-perhaps all others. Many of these disciplines were represented at the Banbury meeting, but not all. In part, this was due to logistical limitations (e.g., limited meeting space or lack of availability of participants for the dates of the meeting). In part, however, the meeting was designed to focus on developing strategies to include the recruitment of physician medical ge-

\footnotetext{
From the ${ }^{1}$ University of Alabama at Birmingham, Birmingham, Alabama; ${ }^{2}$ Wayne State University School of Medicine, Detroit, Michigan; and ${ }^{3}$ Case Western Reserve University, Cincinnati, Ohio.

Bruce Korf, MD, PhD, University of Alabama at Birmingham, Birmingham, AL.
}

DOI: 10.1097/01.GIM.0000171324.58121.CF neticists, with full recognition that physician geneticists will need to work side-by-side with other colleagues, including other genetics professionals and physicians from other specialties. The meeting also did not focus on issues of reimbursement for medical genetic services. It could be argued that increasing reimbursement would quickly attract more people to the discipline and increase the funding to training programs. Although this may be true, achieving this goal will be an arduous and prolonged campaign, especially in the current climate for health care reimbursement. Success will require vigorous advocacy and demonstration of clarity of vision on the part of the medical genetics community. Seeking creative solutions to attract the brightest physicians and other professionals to the genetics community will be critical to addressing the economic needs of the discipline.

\section{STATEMENT OF THE PROBLEMS}

(1) The number of physicians choosing to enter the field of medical genetics is small, and getting smaller. (2) There will be increasing need for physicians who are trained in medical genetics as our understanding of the genetic contribution to rare and common disorders increases. (3) Current medical genetics training does not adequately prepare trainees to be the leaders in the integration of genetics into medical practice.

The roots of medical genetics as a distinct profession can be traced to the early days of the American Society of Human Genetics, which was founded in 1948. At that time, the focus was on rare disorders, particularly in the areas of biochemical genetics, population genetics, and immunogenetics, with gradual additions of clinical genetics and cytogenetics over the ensuing decade. Several training programs came into existence, although during this time there was no formal training path or official recognition of medical genetics as a specialty. The initial training programs were all rooted in adult medicine. During the 1960s and 1970s, rapid advances in cytogenetics and prenatal diagnosis shifted the focus of the profession toward perinatal and pediatric issues. Training in medical genetics was formalized when the American Board of Medical Genetics (ABMG) was founded in 1980. Fellowships were established for clinical genetics, as well for PhDs, laboratory geneticists, and genetic counselors. In 1991, the field gained recognition by admission of the ABMG into the American Board of Medical Specialties (ABMS), the governing organization for all primary medical specialties in the United States. At that same time, the 
American College of Medical Genetics (ACMG) was established. Membership in the ABMS required that medical genetics training be configured as a residency, under the aegis of a residency review committee (RRC) functioning under the umbrella of the Accreditation Council of Graduate Medical Education (ACGME). The Medical Genetics RRC began to accredit residencies in 1995. Clinical genetics fellowships were phased out, replaced by medical genetics residencies.

The number of individuals who achieve certification in clinical genetics has been declining in recent years (Fig. 1). The large number certified in 1982 represents practicing geneticists who took the first examination. After that, numbers remained stable, albeit low compared with other medial specialties, until the transition from fellowship to residency training in 1996. Since then, numbers have been declining through the last exam cycle in 2002. The number of training positions in RRC-accredited programs is correspondingly small, but stable since 1999 (Fig. 2). In 2000, the number of training programs reached a plateau in the mid-40s. Currently, there are 193 approved positions, of which 82 are filled.

Why are the numbers of physicians who seek training in medical genetics declining? Participants at the Banbury meeting were polled, resulting in the list of issues summarized in Table 1. Although not an exhaustive accounting, the same issues were raised repeatedly and independently, suggesting that there are at least several major contributing factors.

These declining numbers occur at a time when the opportunities to integrate genetics into medical practice are advancing dramatically. Areas of traditional interest to medical geneticists such as dysmorphology, prenatal diagnosis, and inborn errors of metabolism, are evolving rapidly. Genes responsible for congenital anomaly syndromes are quickly coming to light; prenatal diagnosis is evolving with the advent of first trimester screening and expanded approaches to carrier screening; newborn screening is being vastly extended with tandem mass spectrometry; treatments such as enzyme replacement therapy are transforming the management of some metabolic disorders.

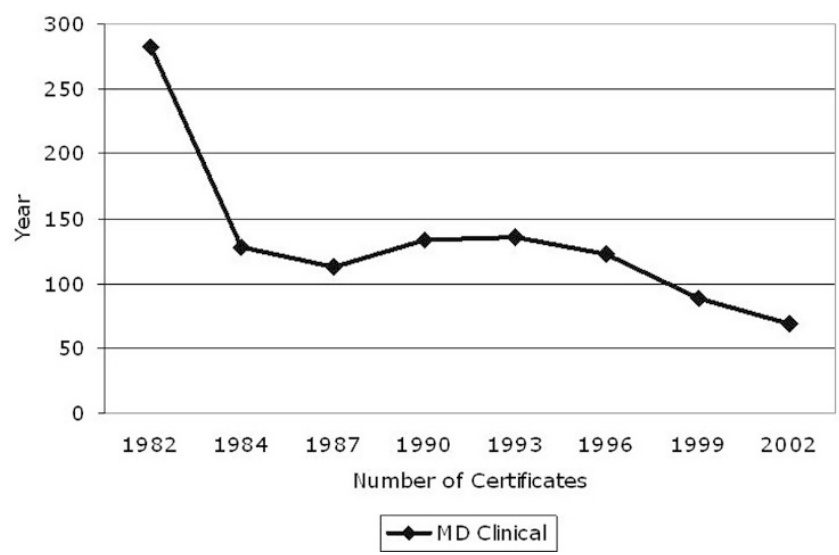

Fig. 1. Number of MD Clinical Genetics certificates issued by the ABMG in each exam year since the initiation of certification in 1982 .

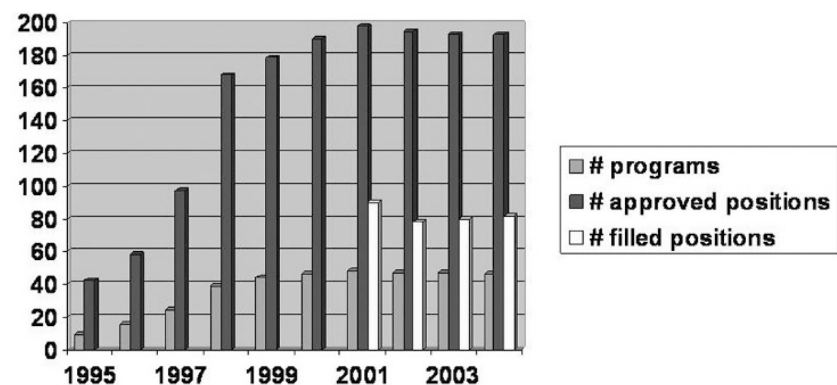

Fig. 2. Number of RRC-approved programs, training slots, and filled slots in medical genetics.

\section{Table 1}

List of major reasons cited by participants at Banbury Summit Meeting $(n=20)$ to explain declining number of physicians who seek training in medical genetics

\begin{tabular}{lc}
\hline $\begin{array}{l}\text { Issue } \\
\begin{array}{l}\text { Lack of awareness of genetics/insufficient exposure in } \\
\text { medical school }\end{array}\end{array}$ & $\begin{array}{c}\text { Percent of } \\
\text { Respondents }\end{array}$ \\
$\begin{array}{l}\text { Uncertain future for genetics: genetics is not regarded as a } \\
\text { primary specialty and not considered part of mainstream } \\
\text { medicine }\end{array}$ & 50 \\
$\begin{array}{l}\text { Low compensation for medical geneticists } \\
\text { Poor reimbursement for clinical genetic services }\end{array}$ & 40 \\
$\begin{array}{l}\text { Lack of clinical role models for medical students and } \\
\text { residents }\end{array}$ & 30 \\
$\begin{array}{l}\text { Lack of funding for training programs } \\
\text { Poor recruitment/marketing }\end{array}$ & 30 \\
$\begin{array}{l}\text { The perception that clinical genetics is not treatment- } \\
\text { oriented }\end{array}$ & 25 \\
$\begin{array}{l}\text { Length of training is too long } \\
\text { Strong bias to dysmorphology \& biochemical genetics in } \\
\text { most training programs is a disincentive to those with } \\
\text { interest in adult issues }\end{array}$ & 25 \\
$\begin{array}{l}\text { Sense/belief that physicians will be able to practice genetic } \\
\text { medicine w/out specialty training }\end{array}$ & 25 \\
\hline
\end{tabular}

Meanwhile, the relevance of genetics to common disorders is becoming increasingly apparent. Genetic risk assessment and testing have become standard for some forms of cancer; genetic testing is available for a number of neurological and cardiovascular disorders; pharmacogenetic testing is being introduced and will likely influence a wide variety of routine medical decisions. It is not clear that current medical genetics training is preparing medical geneticists to be leaders in the integration of genetics into all areas of medical practice. Although exposure to adult genetics and areas such as cancer genetics are expected for all trainees, most programs - and the certification examination - remain rooted in traditional areas of dysmorphology, prenatal diagnosis, and biochemical genetics.

In this state of flux, it is difficult to know the exact number of medical geneticists that will be needed over the next several 
years. Given the diversity of applications of genetics in medicine, it is likely that a wide variety of types of care models will be used, requiring a diverse set of individuals with genetics training. This includes medical geneticists, genetic counselors, laboratory geneticists, physicians in other specialties who will use genetic testing and other tools in their practice, and to some extent, all medical professionals. Study of the genetic workforce and care models is underway, but lacking these data, it is clear that training fewer medical geneticists at a time of major growth in the field will result in a shortage both of practitioners and of leaders and teachers for colleagues in other disciplines.

\section{STATEMENT OF PRINCIPLES}

(1) Medical genetics is a primary medical specialty. (2) There will continue to be a need for "generalist" medical geneticists, who will deal with a broad range of genetic disorders, maintain an identity for genetics within institutions and in organized medicine, and serve as reservoirs of expertise for training. (3) It is in the best interests of the medical genetics community and the public for medical genetics to partner with other specialty groups to integrate genetics into all areas of medicine.

The rapid advance of knowledge and technology in genetics makes it difficult to know how genetics will be integrated across medicine in the coming years. It is likely that no single model will apply to all areas, and that change will come gradually, and at different rates, in different disciplines. Medical geneticists have the opportunity, however, to play a key role in this process, both by providing care as generalists in medical genetics and by partnering with colleagues in other disciplines.

The recognition of medical genetics as a primary medical specialty was a major milestone. It has provided the field a place at the table in discussions of issues regarding reimbursement for medical services and a voice in deliberations about national health care policy on such bodies of the AMA House of Delegates and the AAMC Council of Academic Specialties. It is unusual among medical specialties, however, in a number of ways. Medical genetics does not focus on any one system of the body or on any particular age group. It is one of the only disciplines that includes non-MDs among those who achieve board certification. It provides a wide range of services, including diagnostic, laboratory, counseling, and patient management and treatment.

It is likely that medical geneticists will play distinct roles in delivery of care in different settings. They will continue to be involved in the care of individuals with "single gene" and chromosomal disorders, providing counseling, and to an increasing extent, management and treatment. Medical geneticists will also be involved in the design, implementation, and interpretation of laboratory tests that are based on genetic analysis. It is unlikely, however, that medical geneticists will see all of the patients on whom testing is performed or who may have a family history that contributes to risk of rare or common disorders. For example, it is likely that pharmacogenetic testing will be used for routine medical decision-making by primary care and specialty physicians. Although geneticists may be involved in developing, performing, and interpreting pharmacogenetic tests, they will not see the vast majority of patients on whom these tests are ordered. A dual role will emerge for medical geneticists: as practitioners who will see patients with genetic disorders and as the reservoirs of knowledge and experience who can oversee the deployment of the genetic approach throughout the medical community.

\section{SOLUTIONS}

Just as medical genetics will play a multifaceted role in the future of medicine, the problem of increasing the numbers of physicians who enter the discipline will have to be approached at multiple levels. This includes increased attention to recruitment into the field, strengthening of the core training of medical geneticists, and seeking creative approaches to partnering with other specialties.

\section{Increase recruitment of trainees in medical genetics}

(1) Position medical genetics as an ideal career for medical students who foresee pursuing an academic career. (2) Seek NIH funding to develop centers of excellence in medical genetics training that will nurture trainees to become leaders in academic medical genetics. (3) Enhance the visibility of medical genetics by working with groups that advise medical students and residents on career choice.

Medical geneticists will need to step up efforts to actively recruit physicians to enter the field. Increasing rates of reimbursement for medical genetics services will be of key importance in achieving this goal, but major steps can be taken even as this long-term campaign proceeds. Medical genetics offers significant attractions as a career choice for the student who wishes to pursue an academic career. Technological and intellectual advances have placed major resources in the hands of laboratory investigators, who have an unprecedented opportunity to understand how genes contribute to health and disease. Opportunities to translate knowledge into new diagnostic tests and treatments provide equally powerful resources for clinical investigators. Medical genetics is positioned at the leading edge of medical research, and, as such, is arguably the most exciting place to be for the academically inclined student or resident.

Lack of institutional funding for the support of medical genetics residencies has been a major handicap. Although some institutions have included medical genetics in their graduate medical education (GME) budgets, this is likely to remain an area of difficulty given the small size of the discipline and the shrinking budgets for GME. The argument can be made, however, that medical genetics is a critical intellectual resource, with vast opportunities to develop new knowledge and apply it to improve the health of the population. As such, the field should be able to attract funding from national sources, especially the NIH, but perhaps also foundations, to help support the training of the next generation of medical geneticists. 
Development of a funding mechanism dependent upon grant support creates the risk that training will be concentrated in a relatively small number of centers. In effect, however, this is already occurring due to very limited institutional funding sources. Whether grant support will be concentrated or more widely distributed has yet to be decided, but it is hard to see how increasing the funds available to support training will do anything but help to address the impending crisis in genetics training.

There are, in addition, some relatively simple approaches that can help to increase the visibility of medical genetics as students and residents plan their careers. There are programs, both national and local institutional programs, for advising students on career choice in which medical geneticists can participate. Efforts should continue to increase the exposure of medical students and residents to genetics as a component of their education. Most importantly, medical geneticists must be visible, active, and tireless advocates for genetics education within their institutions, and must provide role models that will attract future trainees.

\section{Strengthen the core training of generalist medical geneticists}

(1) Review the structure of medical genetics training paths and ensure that the length and content of training is appropriate. (2) Develop and disseminate model genetics residency curricula and teaching materials that broaden the base of medical genetics training. (3) Focus the ABMG general examination on fundamental principles of genetics. (4) Encourage the development of departments of genetics and discrete medical genetics clinical services. (5) Consider development of subspecialty fellowships within genetics.

Medical genetics training currently requires a 2 -year residency, but completion of a separate residency of at least 2 years is a prerequisite. The total training time may be as long as 5 years if board certification is sought both in genetics and in the other discipline (e.g., medicine, pediatrics, etc.), and can be further prolonged if research training is included. Perhaps the complexity of the discipline dictates an extended period of training, but there is significant risk that the long time required will deter some from entering the field. The structure of medical genetics training should be reviewed to ensure that the duration of training is appropriate, and neither too long nor too short.

Development and dissemination of curricular models and teaching materials will be helpful, particularly as training programs increase content on the genetics of common and adultonset disorders. As the front of genetics knowledge and its application to medicine advances, programs will benefit from sharing of teaching models to integrate new approaches into their training.

Efforts to broaden the scope of genetics training to include coverage of both rare and common disorders must be coordinated at multiple levels. The certifying exam is administered by the ABMG, whereas programs are accredited by the RRC. Although these are independent bodies, there should be some degree of synchrony between the content of the residency and the content of the examinations. For example, it would not make sense for residencies to increase the content of teaching about the genetics of common disorders if the ABMG continues to emphasize rare disorders. The ABMG examination consists of two parts: a general examination and a specialty examination (e.g., in clinical genetics). The ABMG should insure that the general examination is indeed focused on general principles of genetics and not on the diagnosis and management of rare genetic conditions. This will be especially important if subspecialty tracks are created (see next section), because the general examination can and should serve as a means of assessment of general genetics knowledge and not knowledge of rare disorders.

If medical geneticists are to play a central role in the integration of genetics into medicine, it will be important for them to have a clearly defined platform for their activities in academic medical centers. This includes not only having a context for providing patient care and doing research, but also having a voice in the formulation and execution of institutional policy. The organizational structure of genetics differs in different institutions, comprising divisions within departments, entire departments, centers, etc. Regardless of the specific institutional models, the development of well-delineated clinical and academic units will enhance the ability of medical geneticists to serve as institutional leaders.

Genetics is a broad discipline that relates to virtually every aspect of human health and disease. As the power of the genetic approach comes to be applied across all of medicine, it will become increasingly difficult for the geneticist to be equally adept at all applications. It may be desirable to provide more focused subspecialty tracks for the medical geneticist who wishes to gain special expertise in a specific area. This approach is already beginning to be implemented for training medical geneticists who wish to provide care to patients with inborn errors of metabolism (but who do not wish to pursue the laboratory-based fellowship training in clinical biochemical genetics). Eventually, it may be provided to those who wish to focus on other areas, such as cancer genetics. Such subspecialty options would probably take the form of 1-year fellowships for those who have completed a 2-year genetics residency.

\section{Partner with other medical specialties}

(1) Encourage the development of combined residency programs with other specialties. (2) Pursue joint subspecialty fellowships with other specialties that are highly focused on the genetics of individual disciplines.

Medical geneticists can be the leaders for incorporation of genetics into medical practice, but they cannot accomplish this goal single-handedly. Patients with neurological disorders or cancer will continue to be taken care of by neurologists and oncologists, regardless of whether the underlying causes of their disorders are genetic. We will increasingly encounter physicians who wish to subspecialize in the provision of genetic services within a specific medical discipline. Some of these individuals may wish to pursue the broad-based genetics training that comprises a medical genetics residency, but oth- 
ers will be deterred from doing so by the long period of training required and by the need to devote significant time during training to areas of genetics that are not related to their underlying interest. An oncologist who wishes to become an expert in cancer genetics, for example, may not be prepared to devote significant training time to learning about the care of children with congenital anomalies or inborn errors of metabolism.

Two broad approaches are visualized for the partnership of geneticists with other disciplines. First, there can be joint residency training programs that combine medical genetics with another major discipline. This has already been implemented in the form of combined programs in genetics and pediatrics, internal medicine, and maternal-fetal medicine. Plans are being developed for a combined program with neurology and with family practice. Such combined programs lead to qualifications to take board examinations in genetics and the other discipline. They do not necessarily save time in training, but they do attract students with an interest in genetics who might otherwise pursue training in a more classical area of medicine and permit an early focus on medical genetic aspects of the discipline. Further, combined programs allow for focused biomedical research in genetics and can be used as a vehicle to attract future physician-scientists to develop careers in multidisciplinary research. These programs are in their early stages of implementation, but hold promise for increasing the numbers of individuals trained in medical genetics. The goal, however, remains the provision of broad-based genetics training, albeit with a focus on the intersection of genetics and a major specialty area.

The second approach, which is complementary to the first, is the development of subspecialty fellowships in narrowly defined areas of genetic medicine. These would be 1- or 2-year fellowships that would provide training in a specific application of medical genetics. An example might be cancer genetics, where the training would focus on risk assessment, interpretation of genetic testing, and management of patients found to be at high risk of cancer. The fellowship training could be offered both to medical geneticists and to oncologists. It would qualify a geneticist to counsel cancer patients, but not to treat their cancer; it would qualify an oncologist to counsel cancer patients but not to provide care for other types of genetic disorders. Individuals who complete such training might be required to pass the ABMG general examination, and a discipline-specific examination, and/or present a logbook of relevant experience.

The concept of providing the subspecialty fellowships and opening these to individuals trained outside of genetics might be construed as risking the identity of medical genetics, but also might be seen as the one viable approach to expanding the field of medical genetics to meet the anticipated challenges of integrating genetics across all of medicine. If genetics is to become a component of medical practice for all specialties, this will require training a much larger cadre of practitioners than is feasible for the foreseeable future if all trainees are required to complete a full genetics residency. Moreover, it is very unlikely that geneticists will ever assume full responsibility for the care of all patients who have any genetic contribution to their disorder, because eventually this will be all patients. Other specialties will incorporate genetics into their practice with or without the help of medical geneticists. If the genetics community is prepared to partner with them it can provide a model that will preserve a critical role for the generalist geneticist while facilitating the inclusion of genetics into medicine in a manner that will allow wide dissemination of the genetic approach.

\section{CONCLUSIONS}

Medical genetics training is approaching a crossroads. Extrapolation of the rate of decline in the number of physicians entering the field into the future suggests the possible extinction of the discipline. Even if the numbers stabilize, if nothing is changed, the numbers will be inadequate to meet the demand of deploying genetics across all of medicine. Medical genetics is a relatively young discipline, and has seen a rate of growth of new knowledge almost unprecedented among medical specialties. This rapid pace of change both adds to the excitement and to the challenge of being a practitioner in medical genetics. Now it is also demanding an ability to adapt, so that what may be the smallest medical specialty in terms of numbers can be its most influential in terms of impact on health care.

\section{AFTERWORD}

Since the distribution of this report in January, there have been several opportunities to gather feedback from the community and to move forward on some of the initiatives proposed at the meeting. Written comments were received from members of the American College of Medical Genetics and a portion of the March, 2005 ACMG business meeting was dedicated to the topic. Discussions have also taken place at the meeting of the Association of Professors of Human and Medical Genetics, as well as the board meetings of the participating organizations and at the April Genetics Residency Review Committee meeting. A working group has been organized to carry forward the initiatives proposed at the meeting, which includes leadership from the ACMG, ABMG, ASHG, Genetics RRC, and APHMG.

In reviewing the feedback, several important points have been raised that should be acknowledged here:

- The Banbury Report focused on genetics as an academic discipline, but did not emphasize opportunities for genetics practice in nonacademic settings (including private practice and practice within an HMO). Further discussions of initiatives aimed at increasing the recruitment of physicians in medical genetics will consider both academic and nonacademic settings.

- Although the focus of the Banbury Report was not on the economics of genetics practice, it is clear that this issue will need to be addressed as a component of the solution to increasing physician recruitment to the discipline. 
- There are many areas where it would be helpful to collect additional data, for example, determining the perceptions of medical students about genetics as a career. Efforts will be made to collect additional data on selected issues, but this will be done in parallel with implementation of initiatives to address problems that are already evident.

- The possibility of creating separate pediatrics and adult genetics tracks in residency training has been raised. It may be unwise to further subdivide an already small field, but it is recognized that both training requirements and the $A B M G$ examination need to be better balanced between pediatric and adult genetics issues.

The leadership of the ACMG, ABMG, ASHG, APHMG, and RRC remain open to additional suggestions and comments, but will now be focusing on moving forward with many of the recommendations that have emerged from the meeting and the subsequent feedback.

\section{APPENDIX 1}

\section{Banbury Summit meeting participants}

Meeting Organizer: Bruce R. Korf, MD, PhD, University of Alabama at Birmingham. Meeting Coorganizers: Gerald Feldman, $\mathrm{MD}, \mathrm{PhD}$, Wayne State University School Medicine; Georgia L. Wiesner, MD, Case Western Reserve University. Association of Professors of Human and Medical Genetics (APHMG): Reed Pyeritz, MD, PhD, University Pennsylvania School Medicine; Gerald Feldman, MD, PhD, Wayne State University School Medicine. Accreditation Council for Graduate Medical Education (ACGME): Steven P. Nestler, PhD, ACGME; Douglas Riegert-Johnson, MD, Johns Hopkins University School Medicine. Medical Genetics (ABMG): Judith Cooksey, MD, MPH, University Maryland-Baltimore; Georgia L. Wiesner, MD, Case Western Reserve University. American Board of American Academy of Pediatrics (AAP): Virginia Proud, MD, Eastern Virginia Medical School. American College of Medical Genetics (ACMG): Charles J. Epstein, MD, University California; Marilyn C. Jones, MD, Children's Hospital, University California, San Diego; Michael S. Watson, PhD, ACMG. American Society of Human Genetics (ASHG): Joann A. Boughman, PhD, ASHG; Robert L. Nussbaum, MD, NHGRI/NIH; Peter H. Byers, MD, University Washington. American Board of Genetic Counseling (ABGC): Elsa W. Reich, MS, New York University School Medicine; Anne E. Greb, MS, Wayne State University School Medicine. National Society of Genetic Counselors (NSGC): Maureen Smith, MS, CGC, Northwestern University. Canadian College of Medical Geneticists (CCMG): D. Ross McLeod, MD, Alberta Chil- dren's Hospital. National Human Genome Research Institute (NHGRI): William Gahl, MD, PhD, NHGRI/NIH. Society for Inherited Metabolic Disorders: Mendel Tuchman, MD, Children's National Medical Center (SIMD); Jerry Vockley, MD, $\mathrm{PhD}$, Children's Hospital, Pittsburgh. Royal College of Physicians and Surgeons of Canada: Gail E. Graham, MD, Children's Hosp Eastern Ontario. Additional Participants: Lynn Fleisher, PhD, Sidley Austin Brown \& Wood LLP; Amy E. Roberts, MD. Former resident representative Medical Genetics RRC, Harvard-Partners Center for Genetics and Genomics.

\section{APPENDIX 2}

\section{Glossary of organizations}

American Board of Medical Genetics (ABMG): responsible for certification of medical geneticists; in addition serves both as accrediting body for training programs and certifying organization for clinical cytogenetics, clinical molecular genetics, and biochemical genetics. American Board of Medical Specialties (ABMS): organization that coordinates the activity of 24 approved medical specialty boards in the United States. American College of Medical Genetics (ACMG): professional society representing medical genetics, consisting of biochemical, clinical, cytogenetic, medical and molecular geneticists, genetic counselors, and other health care professionals committed to the practice of medical genetics. Accreditation Council of Graduate Medical Education (ACGME): responsible for accreditation of post-MD training programs in the United States. American Medical Association House of Delegates: principal policymaking body of the American Medical Association, consisting of elected delegates and appointees of specialty societies; ACMG has one seat in the House of Delegates. American Society of Human Genetics (ASHG): professional membership organization of human geneticists in the Americas. Association of American Medical Colleges Council of Academic Societies (AAMC CAS): one of three governing councils of the AAMC, composed of 96 academic societies; genetics is represented by the ACMG Foundation, ASHG, and APHMG. Association of Professors of Human and Medical Genetics (APHMG): organization of medical school professors of genetics, chairs, and program directors. Graduate Medical Education (GME): post-MD medical training (usually residency education). Medical Genetics Residency Review Committee (Medical Genetics RRC): responsible for accreditation of medical genetics residency programs; operates under the ACGME. 